TRANSACTIONS OF THE

AMERICAN MATHEMATICAL SOCIETY

Volume 235, January 1978

\title{
BASES FOR THE POSITIVE CONE OF A PARTIALLY ORDERED MODULE( $\left(^{1}\right)$
}

BY

\author{
W. RUSSELL BELDING
}

\begin{abstract}
R, R^{+}\right)$is a partially ordered ring and $\left(M, M^{+}\right)$is a strict $\left(R, R^{+}\right)$-module. So $M$ is a left $R$-module and $\left(R^{+} \backslash\{0\}\right)\left(M^{+} \backslash\{0\}\right) \subseteq$ $M^{+} \backslash\{0\}$. Let $<^{\prime}$ be the order induced on $M$ by $M^{+} . B \subseteq M^{+}$is an $R^{+}$-basis for $M^{+}$means $R^{+} B=M^{+}$(spanning) and if $r$ is in $R, b$ in $B$ with $0<' r b<' b$ then $r b \notin R^{+}(B \backslash\{b\})$ (independence).

Result: If $B$ and $D$ are $R^{+}$-bases for $M^{+}$then card $B=$ card $D$ and to within a permutation $b_{i}=u_{i} d_{i}$ for units $u_{i}$ of $R^{+}$.
\end{abstract}

Introduction. $\mathrm{A}$ closure operator $c$ on a set $X$ is a map from the subsets of $X, \mathcal{P}(X)$, to $\mathcal{P}(X)$ with the following properties: $A \subseteq c A ; c A=c c A$ and $A \subseteq B$ implies $c A \subseteq c B$. The closure operator $c$ is algebraic if $c A=\cup c A_{0}$ where $A_{0}$ ranges over the finite subsets of $A$. Sets of the form $c A$ are closed and the closed sets form a complete lattice under $\bigvee c A_{i}=c\left(\cup A_{i}\right)$ and $\wedge c A_{i}=\cap c A_{i}$. A set $B$ is a basis for $c A$ if $c B=c A(B$ spans $c A)$ and $b \in B$ implies $b \notin c(B \backslash\{b\})$ ( $B$ is independent). The closure operator $c$ has the exchange property if $a \notin c A$ and $a \in c(A \cup\{b\})$ implies $b \in c(A \cup\{a\})$. It is known (Cohn [3, Chapter VII]) that for an algebraic closure operator $c$ with the exchange property the following statements are equivalent: $B$ is a basis for $c A ; B$ is a maximal independent subset of $c A ; B$ is a minimal spanning set for $c A$. Further, every closed set $c A$ has a basis and two bases for $c A$ have the same cardinality. These results may fail if $c$ does not have the exchange property.

The simplest example of an algebraic closure operator having a closed set with bases of distinct cardinalities is the following (M. N. Bleicher and E. Marczewski [1, p. 211]): $X=\{1,2,3\}$ and $S \subseteq X$

\begin{tabular}{c|c|c|c|c|c|c|c|c|}
$S$ & $\varnothing$ & 1 & 2 & 3 & 12 & 13 & 23 & $X$ \\
\hline$c S$ & $\varnothing$ & 1 & 2 & $X$ & $X$ & $X$ & $X$ & $X$
\end{tabular}.

Received by the editors February 12, 1976 and, in revised form, June 7, 1976.

AMS (MOS) subject classifications (1970). Primary 06A70, 06A10.

Key words and phrases. Partially ordered ring, partially ordered module, directed, order preserving automorphism group, closure operator.

$\left.{ }^{1}\right)$ This work was supported by a grant from the Naval Academy Research Council. 
Observe that $\{3\}$ and $\{1,2\}$ are bases for $X$. Example 5, below, gives an example of a maximal independent set $B \subseteq c A$ which is not a basis for $c A$. Yet, in the examples of Theorem 3, every closed set has a unique basis. So the exchange property is sufficient but not necessary for every closed set to have a basis and for bases to have the same cardinality.

The following problems naturally arise for algebraic closure operators. Find necessary and sufficient conditions for a closed set to have a basis. Find necessary and sufficient conditions for bases of a closed set to have the same cardinality. This paper is about sufficient conditions for bases to have the same cardinality for one class of closure operators. For this class of closure operators we need to strengthen the definition of independence to get this result.

1. Definitions. We state some standard definitions which are found in $L$. Fuchs [4] and P. Ribenboim [7]. A partially ordered group $\left(G,<^{\prime}\right)$ has $<^{\prime}$ as a partial order on $G$ and if $a \leqslant^{\prime} b$ then for every $u, v$ in $G, u a v \leqslant^{\prime} u b v$. Equivalently we could write $\left(G, G^{+}\right)$and require the positive cone $G^{+}$to satisfy: reflexivity, $1 \in G^{+}$; antisymmetry, $G^{+} \cap\left(G^{+}\right)^{-1}=\{1\}$; transitivity, $G^{+} G^{+} \subseteq G^{+}$; and monotonicity, for $g$ in $G, g G^{+} g^{-1} \subseteq G^{+}$. If $<^{\prime}$ is the order induced on $G$ by $G^{+}$then $G^{+}=\left\{g \mid 1<^{\prime} g\right\} .\left(G, G^{+}\right)$is directed if every $g$ in $G$ can be written $x y^{-1}$ for some $x, y$ in $G^{+}$; this is equivalent to every pair $\{g, h\}$ in $G$ having an upper and a lower bound in $G$. A partially ordered ring $\left(R, R^{+}\right)$is a partially ordered group with respect to addition and $R^{+} R^{+} \subseteq R^{+}$. The ordering is strict if $\left(R^{+} \backslash\{0\}\right)\left(R^{+} \backslash\{0\}\right) \subseteq R^{+} \backslash\{0\}$. Should the order induced by $R^{+}$not be strict, let $D_{0}=\left\{x \in R^{+} \mid x \neq 0 \& \exists y\right.$ $\left.\left(y \in R^{+} \& y \neq 0 \& x y=0\right)\right\}$ and $R^{\#}=R^{+} \backslash D_{0}$; then the partially ordered ring $\left(R, R^{\#}\right)$ has a strict ordering. For example, let $C=C[0,1]$ be the ring of continuous maps from $[0,1]$ to the reals, $\mathbf{R}$. Let $C^{+}$consists of those $f$ in $C$ such that $0<f(x)$ for all $x$ in $[0,1]$. The ordering induced by $C^{+}$on $C$ is not strict. $C^{\#}$ consists of those $f$ in $C^{+}$such that $f^{-1}\{0\}$ contains no nonempty open interval $(a, b) \subseteq[0,1]$.

If $\left(M, M^{+}\right)$is a partially ordered group it is also an $\left(R, R^{+}\right)$-module if $M$ is a left $R$-module and if $R^{+} M^{+} \subseteq M^{+}$. It is a strict $\left(R, R^{+}\right)$-module if $\left(R^{+} \backslash\{0\}\right)\left(M^{+} \backslash\{0\}\right) \subseteq M^{+} \backslash\{0\}$. We restrict ourselves to strict $\left(R, R^{+}\right)$modules. In particular if $r \in R^{+}, m \in M^{+} \backslash\{0\}$ and $r m=0$ then $r=0$. Consequently the ordering induced by $R^{+}$on $R$ must be strict. We require rings to have a 1. The units of $R^{+}$are the units $u$ of $R$ for which both $u$ and $u^{-1}$ are in $R^{+}$. In particular a unit of $R^{+}$is more than a unit of $R$ which is in $R^{+}$. The nonnegative integers, rationals and reals are written $Z^{+}, Q^{+}, \mathbf{R}^{+}$ respectively.

EXAMPLE 1. A partially ordered abelian group $\left(M, M^{+}\right)$is a strict $\left(Z, Z^{+}\right)$-module as the positive cone $M^{+}$is torsion free. 
What seem to be natural requirements in the proofs of Theorems 1 and 2 lead to a modification of the definition of a basis for closure operators on abelian groups. Let $M$ be an abelian group and $c$ a closure operator on $M$. Let $B \subseteq c A \subseteq M$. Then $B$ is a basis for $c A$ if (i) $c B=c A$ and (ii) $a \in M$, $b \in B, a \in c\{b\}$ and $b-a \in c B$ implies $a \notin c(B \backslash\{b\})$. So only the notion of independence is changed from the standard definition given earlier. If $B$ is independent in this new sense it is independent in the standard sense but not conversely. From now on we use only this new definition.

2. $R^{+}$-bases for $M^{+}$. Let $\left(R, R^{+}\right)$be a partially ordered ring and $M$ an $R$-module. If $B \subseteq M$ let $c B$ be the set of finite sums $\Sigma r_{i} b_{i}$ with $b_{i}$ in $B$ and $r_{i}$ in $R^{+}$. Then $c$ is an algebraic closure operator on $M$. For convenience we write $R^{+} B=c B$. If $\left(M, M^{+}\right)$is a strict $\left(R, R^{+}\right)$-module and $B \subseteq M^{+}$then $\left(M, R^{+} B\right)$ is a strict $\left(R, R^{+}\right)$-module. The set $B R^{+}$-spans $M^{+}$if $R^{+} B=$ $M^{+}$. If $<^{\prime}$ is the order induced by $M^{+}$on $M$ then $B$ is $R^{+}$-independent if for $b$ in $B$ and $r$ in $R$ we have $0<^{\prime} r b<^{\prime} b$ implies $r b \notin R^{+}(B \backslash\{b\})$. With both these properties, $B$ is an $R^{+}$-basis for $M^{+}$. This is how the new definition of basis is interpreted in this context.

EXAMPLE 2. Let $P_{n}=\left\{0, x_{1}, x_{2}, \ldots, x_{n}=1\right\}$ be any partition of $[0,1]$ and let $f_{k} \in C$ be nonzero only on $\left(x_{k-1}, x_{k}\right)$ and nonnegative on this interval. Then $B=\left\{f_{1}, \ldots, f_{n}\right\} \subseteq C^{+} \backslash C^{\#}$ but $\left(C, C^{\#} B\right)$ is a strict $\left(C, C^{\#}\right)$-module.

Let $<$ ' be the order induced on $C$ by $C^{\#} B$. If $g \in C$ and $0<{ }^{\prime} g f_{k}<f_{k}$ it is straightforward to check that $g f_{k} \notin C^{\#}\left(B \backslash\left\{f_{k}\right\}\right)$. Clearly the members of $C C^{\#} B$ do not have unique expressions as $C^{\#}$-linear sums of the $f_{k}$.

EXAMPLE 3. Let $I$ be an index set and $\left(R, R^{+}\right)$a partially ordered ring. Then $\bigoplus_{I}\left(R, R^{+}\right)=\left(\bigoplus_{I} R, \bigoplus_{I} R^{+}\right)$, having the product ordering $\left(r_{i}\right)<^{\prime}\left(s_{i}\right)$ iff $r_{i}<s_{i}$ for each $i$, is an $\left(R, R^{+}\right)$-module. The cone $\bigoplus_{I} R^{+}$has $R^{+}$-basis $\left\{\left(\delta_{i j}\right)_{j \in I} \mid i \in I\right\}$ where $\delta_{i j}$ is the Kronecker delta in $R$.

EXAMPLE 4. Let $B$ be a set of positive reals which are independent as vectors over $Q$. Then $B$ is a $Q^{+}$-independent set, $\left(\mathbf{R}, Q^{+} B\right)$ is a $\left(Q, Q^{+}\right)$module and $B$ is a $Q^{+}$-basis for the cone $Q^{+} B$. The ordering $<'$ induced by $Q^{+} B$ on $\mathbf{R}$ is $x<{ }^{\prime} y$ iff $y-x \in Q^{+} B$.

By the definition of $R^{+}$-independence, a set $B \subseteq M^{+}$is $R^{+}$-independent if and only if each finite subset of $B$ is $R^{+}$-independent. So the union of a chain of $R^{+}$-independent subsets of $M^{+}$is an $R^{+}$-independent subset. Zorn's lemma gives that each $R^{+}$-independent set $B \subseteq M^{+}$can be extended to a maximal $R^{+}$-independent set $B_{m}, B \subseteq B_{m} \subseteq M^{+}$. Like the extension of independent sets of vectors, $B_{m}$ is not unique. Unlike the extension of independent sets for algebraic closure operators with the exchange property, it is not necessary that $B_{m} R^{+}$-span $M^{+}$.

Example 5. $\left(Z, Z^{+}\right)$is a $\left(Z, Z^{+}\right)$-module. $\{2\}$ is a $Z^{+}$-independent set 
and for every $k \geq 1,\{2,2 k+1\}$ is a maximal $Z^{+}$-independent subset of $Z^{+}$. Of these sets $\{2,3\}$ has the largest $Z^{+}$-span, $Z^{+}\{2,3\}=Z^{+} \backslash\{1\}$.

Lemma 1. If $\left(M, M^{+}\right)$is an $\left(R, R^{+}\right)$-module and $M^{+}$has an $R^{+}$-basis $B$ then $1 \in R^{+}$.

Proof. Let $\xi^{\prime}$ be the order induced on $M$ by $M^{+}$. Let $b \in B$ and $b=\sum r_{i} b_{i}$ for some $b_{i}$ in $B$ and $r_{i}$ in $R^{+}$; then $b$ must appear in the sum by the $R^{+}$-independence of $B$. If $b=b_{j}$ then $0 \leqslant{ }^{\prime}\left(1-r_{j}\right) b=\sum_{i \neq j} r_{i} b_{i} \leqslant{ }^{\prime} b$. By $R^{+}$-independence $\left(1-r_{j}\right) b=0$ and by strictness of the order, $1=r_{j}$; so $1 \in R^{+}$.

It is natural to ask, in the general case, how two $R^{+}$-bases for $M^{+}$are related. If $M^{+}$has $R^{+}$-bases $B$ and $D$ then $B$ and $D$ have the same cardinality and to within a permutation $b_{i}=u_{i} d_{i}$ for units $u_{i}$ of $R^{+}$.

Theorem 1. If $M^{+}$has $R^{+}$-bases $B=\left\{b_{1}, \ldots, b_{m}\right\}$ and $D=\left\{d_{1}, \ldots, d_{n}\right\}$ then $n=m$ and to within a permutation $b_{i}=u_{i} d_{i}$ for units $u_{i}$ of $R^{+}$.

PROOF. For $x_{k j}, y_{j k}$ in $R^{+}, b_{i}=\sum_{j=1}^{n} x_{i j} d_{j}$ and $d_{j}=\sum_{k=1}^{m} y_{j k} b_{k}$. Put $X=$ $\left(x_{k j}\right), Y=\left(y_{k j}\right)$. Let $H=X Y$, then $b_{i}=\sum_{k=1}^{m} h_{i k} b_{k}$. If $<^{\prime}$ is the order induced by $M^{+}$on $M$ then $0 \leqslant{ }^{\prime}\left(1-h_{i i}\right) b_{i}=\Sigma_{k \neq i} h_{i k} b_{k}$. By the $R^{+}$-independence of $B$ and the strictness of the ordering $h_{i i}=1$ and $h_{i k}=0$ for $i \neq k$. Thus $X Y=I_{m}$, the $m$ by $m$ identity matrix over $R$. Similarly $Y X=I_{n}$. So

$$
\sum_{j=1}^{m} x_{i j} y_{j k}=\delta_{i k}=\sum_{j=1}^{n} y_{i j} x_{j k} .
$$

Each $b_{i}$ is nonzero so $x_{i j} \neq 0$ for some $j$; so each row of $X$ has at least one nonzero entry. If for a fixed $j, x_{i j}=0$ for all $i$ then the $b_{i}$ can be written as $R^{+}$-linear sums of $D \backslash\left\{d_{j}\right\}$. By writing $d_{j}$ as an $R^{+}$-linear sum of the $b_{i}$ we obtain $d_{j} \in R^{+}\left(D \backslash\left\{d_{j}\right\}\right)$, contradicting the $R^{+}$-independence of $D$. Thus $x_{i j} \neq 0$ for some $i$ and each column of $X$ has at least one nonzero entry. Similarly each row and column of $Y$ has at least one nonzero entry. Let $<"$ be the order induced on $R$ by $R^{+}$. Suppose $0<$ " $x_{i p} y_{p i}$ in $R^{+}$. If $k \neq i$ then (1) implies $0 \leqslant$ " $x_{k p} y_{p i}<" 0$ so $x_{k p}=0$ by the strictness of the order $\leqslant$ ". So the $p$ th column of $X$ has precisely one nonzero entry. Similarly each row of $X$ has precisely one nonzero entry. If $X$ has more rows than columns then $X$ has a zero row which is not possible so $m<n$. $X$ has no zero columns so $n<m$, so $m=n$. Similarly each row and column of $Y$ has precisely one nonzero entry. There is a permutation $\sigma$ of $\{1,2, \ldots, n\}$ such that $x_{i \sigma(i)} y_{\sigma(i) i}=1$. To within a permutation, $b_{i}=u_{i} d_{i}$ for units $u_{i}$ of $R^{+}$.

If $B$ and $D$ are $R^{+}$-bases for $M^{+}$and one is finite it is straightforward to show the other is finite. If both $B$ and $D$ are infinite then card $B=$ card $D$ by Proposition 5.5, p. 82 of P. M. Cohn [3] ( $B$ and $D$ are minimal $R^{+}$-spanning 
sets for $M^{+}$and $\left.J_{\Omega}(B)=R^{+} B\right)$. Alternatively, the argument used to show invariance of dimensionality of infinite dimensional vector spaces (N. Jacobson [6, pp. 240-241]) may be applied here without much change, to get card $B=\operatorname{card} D$.

THEOREM 2. Let $\kappa$ be an infinite cardinal and suppose $M^{+}$has $R^{+}$-bases $B=\left\{b_{\alpha} \mid \alpha<\kappa\right\}$ and $D=\left\{d_{\alpha} \mid \alpha<\kappa\right\}$. To within a permutation, $b_{\alpha}=u_{\alpha} d_{\alpha}$ for units $u_{\alpha}$ of $R^{+}$.

Proof. For integers $n(\alpha), m(\beta)$ let

$$
b_{\alpha}=\sum_{j=1}^{n(\alpha)} x_{\alpha \beta(j)} d_{\beta(j)} \text { and } \quad d_{\beta}=\sum_{k=1}^{m(\beta)} y_{\beta \gamma(k)} b_{\gamma(k)} .
$$

$x_{\alpha \beta}, y_{\beta \gamma}$ are in $R^{+}$. For $\alpha, \beta<\kappa$ let $x_{\alpha \beta}=0$ if $x_{\alpha \beta}$ does not appear in the sum for $b_{\alpha}$; also put $y_{\beta \gamma}=0$ if it does not appear in the sum for $d_{\beta}$. Let $X=\left(x_{\alpha \beta}\right)$, $Y=\left(y_{\alpha \beta}\right)$ with entries in $R^{+}$. Each row of $X$ and $Y$ has finitely many nonzero entries so the products $X Y, Y X$ exist. As in the proof of Theorem 1 , $X Y=I=Y X$ and each row and column of $S$ and $Y$ has precisely one nonzero entry. The result follows as in the previous theorem.

In view of Theorems 1 and 2 we may define the degree of $M^{+}$over $R^{+}$, $d\left(M^{+}, R^{+}\right)$, to be the cardinality of any $R^{+}$-basis for $M^{+}$, if such a basis exists. If $\left(R_{1}, P_{1}\right)$ is an $\left(R_{2}, P_{2}\right)$-module, $\left(R_{2}, P_{2}\right)$ an $\left(R_{3}, P_{3}\right)$-module, $\left(R_{1}, P_{1}\right)$ an $\left(R_{3}, P_{3}\right)$-module and each degree is defined, it is straightforward to check that $d\left(P_{1}, P_{3}\right)<d\left(P_{1}, P_{2}\right) d\left(P_{2}, P_{3}\right)$. Equality does not have to hold.

Example 6. $\left(R_{3}, P_{3}\right)$ is $\left(Z, Z^{+}\right) ;\left(R_{2}, P_{2}\right)$ is $\left(\mathbf{R}, Z^{+}\{1, \sqrt{3}, \sqrt{5}\}\right)$ and $\left(R_{1}, P_{1}\right)$ is $\left(\mathbf{R}, P_{2}\{1, \sqrt{6} ; \sqrt{10}\}\right)$. Then $\{1, \sqrt{3}, \sqrt{5}\}$ is a $P_{3}$-basis for $P_{2}$ and $\{1, \sqrt{6}, \sqrt{3}\}$ is a $P_{2}$-basis for $P_{1}$. However, $\{1, \sqrt{3}, \sqrt{5}, \sqrt{6}, 3 \sqrt{2}$, $\sqrt{30}, 5 \sqrt{2}\}$ is a $P_{3}$-basis for $P_{1}$. Every cone $Z_{c}$, with $\left(Z, Z_{c}\right)$ a partially ordered group, may be characterized by a unique $Z^{+}$-basis.

THEOREM 3. If $\left(Z, Z_{c}\right)$ is a partially ordered group then $Z_{c}$ has a unique and finite $Z^{+}$-basis.

Proof. $\leqslant$ ' is the order induced by $Z_{c}$ on $Z$. The symbol $<$ and the terms least, positive and negative all refer to the usual ordering in $Z$. Observe that if $Z_{c}$ contains positive and negative integers then $Z_{c}=m Z$ for some integer $m$ and $\zeta^{\prime}$ is not antisymmetric.

First suppose $Z_{c} \subseteq Z^{+}$and let $n_{1}$ be the least member of $Z_{c}$. Let $n_{2}$ be the least member of $Z_{c} \backslash Z^{+}\left\{n_{1}\right\}$ if this set is nonempty, Generally let $n_{k}$ be the least member of $Z_{c} \backslash Z^{+}\left\{n_{1}, \ldots, n_{k-1}\right\}$ if this set is nonempty, otherwise the procedure stops. Since $n_{2}$ must lie between $t n_{1}$ and $(t+1) n_{1}$ for some integer $t$, and so on for the choices $n_{i}$, this procedure will stop in $k<n_{1}$ steps. If $n_{k}$ is the final choice then $Z_{c} \subseteq Z^{+} B$ where $B=\left\{n_{1}, \ldots, n_{k}\right\}$. Since $Z_{c}$ is closed 
under addition and $B \subseteq Z_{c}$ then $Z^{+} B \subseteq Z_{c}$ so $Z_{c}=Z^{+} B$ and $B Z^{+}$-spans $Z_{c}$. If $B$ is not $Z^{+}$-independent then $n_{i} \in Z^{+}\left(B \backslash\left\{n_{i}\right\}\right)$ for some $n_{i}$ in $B$ and $i<k$. According to the procedure $n_{i} \notin Z^{+}\left\{n_{1}, \ldots, n_{i-1}\right\}$ so $n_{i}=m_{t} n_{t}+$ positive terms, for at least one $t>i+1$ and $m_{t}>1$. So $n_{t}<n_{i}$, which is forbidden by the procedure. Thus $B$ is $Z^{+}$-independent and $B$ is a $Z^{+}$-basis for $Z_{c}$. The uniqueness of $B$ follows from Theorem 1 .

If $Z_{c} \subseteq-Z^{+}$then $-Z_{c} \subseteq Z^{+}$and $-Z_{c}$ has $Z^{+}$-basis $B$. So $-B$ is a $Z^{+}$-basis for $Z_{c}$.

The proof given simply picks out the set of atoms $B$ of the ordering $<$ '. So the $Z^{+}$-basis $B$ is the subset of $Z_{c}$ consisting of those elements which sit immediately above 0 in the relation $<^{\prime}$. The proof uses the facts that $\left(Z, Z^{+}\right)$is totally ordered and that $Z^{+}$is well ordered and these two properties characterize the integers. If $\left(R, R^{+}\right)$is a totally ordered ring and $R^{+}$is well ordered, then to within an isomorphism $\left(R, R^{+}\right)=\left(Z, Z^{+}\right)$. (G. A. Heuer [5, p. 135].) M. N. Bleicher and H. Schneider [2, p. 241, Theorem 4.3] have also given a characterization of cones in $Z$.

3. Directed modules and automorphisms. If $\left(R, R^{+}\right)$is directed then every $r$ in $R$ has expression $r=x-y$ for some $x$ and $y$ in $R^{+}$. So $R=R^{+}-R^{+}$. If $\left(M, M^{+}\right)$is directed then $M=M^{+}-M^{+}$. Generally, the directed component of 0 in $R$ is $R_{0}=R^{+}-R^{+}$and the directed component of 0 in $M$ is $M_{0}=M^{+}-M^{+} . R_{0}$ is a subring of $R .\left(M_{0}, M^{+}\right)$is an $\left(R_{0}, R^{+}\right)$-module; for if $r_{1}, r_{2} \in R^{+}, m_{1}, m_{2} \in M^{+}$then $\left(r_{1}-r_{2}\right)\left(m_{1}-m_{2}\right)=\left(r_{1} m_{1}+r_{2} m_{2}\right)-$ $\left(r_{2} m_{1}+r_{1} m_{2}\right)$ is in $M_{0}$. However, $M_{0}$ is not always an $R$-module. $M_{0}$ is also a convex submodule of $M$ if $m_{1}, m_{2} \in M_{0}$ and $m_{1}<' m<{ }^{\prime} m_{2}$ in $M$ then $m \in M_{0}$. Let $m_{1}=a-b$ with $a, b \in M^{+}$. Then $m_{1}<$ ' $m$ implies $0<<^{\prime} a<$ ' $m+b$ so $m=(m+b)-b \in M_{0}$. Hence the quotient module $M / M_{0}$ may be given the induced ordering with positive cone $\left(M / M_{0}\right)^{+}=\left\{m+M_{0} \mid m_{1}\right.$ $\in M^{+}$for some $\left.m_{1} \in m+M_{0}\right\}$. If $m+M_{0} \in\left(M / M_{0}\right)^{+}$then $m \in M_{0}$. So $\left(M / M_{0}\right)^{+}=\{0\}$ and $M / M_{0}$ is trivially ordered. Hence $M$ is an extension of the directed module $M_{0}$ by the trivially ordered module $M / M_{0}$. From now on we assume that rings $\left(R, R^{+}\right)$and their modules $\left(M, M^{+}\right)$are directed. Then, if $M^{+}$has $R^{+}$-basis $B$ we have $M=M^{+}-M^{+}=\left(R^{+}-R^{+}\right) B=$ $R B$.

It is natural to ask for a characterization of those $\left(R, R^{+}\right)$-modules $\left(M, M^{+}\right)$for which $M^{+}$has an $R^{+}$-basis. Let $\bigoplus_{\kappa} R$ denote the direct sum of $\kappa$ copies of $R$ as in Example 3. Let $i_{\beta}$, mapping $R^{\kappa}$ onto the $\beta$ th component in $\bigoplus_{\kappa} R$, be the canonical injection map.

TheOREM 4. The $\left(R, R^{+}\right)$-modules $\left(M, M^{+}\right)$for which $M^{+}$has degree $\kappa$ over $R^{+}$correspond bijectively to the convex $R$-submodules $K$ of $\left(\bigoplus_{\kappa} R\right.$, $\bigoplus_{\kappa} R^{+}$) with the following property: Let $<^{\prime}$ be the ordering induced on $\bigoplus_{\kappa}{ }_{\kappa} R$ 
by $\oplus_{\kappa} R^{+}$. If $r_{\alpha} \in R^{+}$for $\alpha<\kappa$ with only finitely many nonzero and if $s_{\beta} \in R$ and $0<{ }^{\prime} i_{\beta}\left(s_{\beta}\right)<{ }^{\prime} i_{\beta}(1)$ then

$$
i_{\beta}\left(s_{\beta}\right) \neq \sum_{\alpha \neq \beta} i_{\alpha}\left(r_{\alpha}\right) \bmod K
$$

Proof. Suppose $M^{+}$has $R^{+}$-basis $B=\left\{b_{\alpha} \mid \alpha<\kappa\right\}$. Let $f:\left(\bigoplus_{\kappa} R, \bigoplus_{\kappa} R^{+}\right)$ $\rightarrow\left(M, M^{+}\right)$be $f\left(r_{\alpha}\right)=\Sigma r_{\alpha} b_{\alpha}$. As $M=R B, f$ is onto. Clearly $f$ is an $R$-module homomorphism. As $f\left(\oplus_{k} R^{+}\right) \subseteq M^{+}, f$ is order preserving. $K=\operatorname{ker} f=$ $\left\{\left(r_{\alpha}\right) \mid \sum r_{\alpha} b_{\alpha}=0\right\}$. Clearly $K$ is convex in the ordering <' induced by $\bigoplus_{\kappa} R^{+}$ on $\bigoplus_{\alpha} R$. If $0<{ }^{\prime} i_{\beta}\left(s_{\beta}\right)<i_{\beta}(1)$, then $0<{ }^{\prime} s_{\beta} b_{\beta}<b_{\beta}$ (in $M^{+}$) and the $R^{+}$-independence of $B$ gives $s_{\beta} b_{\beta} \neq \Sigma_{\alpha \neq \beta} r_{\alpha} b_{\alpha}$ for $r_{\alpha}$ in $R^{+}$. Thus

$$
i_{\beta}\left(s_{\beta}\right) \neq \sum_{\alpha \neq \beta} i_{\alpha}\left(r_{\alpha}\right) \bmod K \text {. }
$$

Conversely suppose that $K$ is such a convex $R$-submodule of $\bigoplus_{\kappa} R$. Let $P=\left\{\left(r_{\alpha}\right)+K \mid\left(s_{\alpha}\right) \in \bigoplus_{\kappa} R^{+}\right.$for some $\left(s_{\alpha}\right)$ in $\left.\left(r_{\alpha}\right)+K\right\}$. Since $\left(R, R^{+}\right)$is directed each $\left(x_{\alpha}\right)$ in $\bigoplus_{\alpha} R$ can be written $\left(x_{\alpha}\right)=\left(u_{\alpha}\right)-\left(v_{\alpha}\right)$ for some $u_{\alpha}, v_{\alpha}$ in $R^{+}$. Consequently $\left(\bigoplus_{\kappa} R / K, P\right)$ is a directed $\left(R, R^{+}\right)$-module. The given property for $K$ ensures that $\left\{i_{\alpha}(1)+K \mid \alpha<\kappa\right\}$ is an $R^{+}$-basis for $P$.

To show that this correspondence is bijective it is enough to observe that the $R$-module homomorphisms $g$ and $g^{-1}$ where $g:\left(\oplus_{k} R / K, P\right) \rightarrow$ $\left(M, M^{+}\right)$is given by $g\left(\left(r_{\alpha}\right)+K\right)=\Sigma r_{\alpha} b_{\alpha}$ are order-preserving $R$-module isomorphisms.

Suppose that $M^{+}$has $R^{+}$-basis $B=\left\{b_{\alpha} \mid \alpha<\kappa\right\}$. If $f:\left(M, M^{+}\right) \rightarrow$ $\left(M, M^{+}\right)$is an order-preserving $R$-module automorphism it is straightforward to show that $f(B)$ is also an $R^{+}$-basis for $M^{+}$. Using this result we can characterize the group $A$ of order-preserving $R$-module automorphisms of $\left(M, M^{+}\right)$. For convenience let $\left(M, M^{+}\right)$be written as $(\oplus R / K, P)$ as in the previous theorem ( $\kappa$ is fixed so we omit the notation $\bigoplus_{\kappa}$ ). Let $G$ be the group of $\kappa$ by $\kappa$ matrices $X$ having precisely one nonzero entry in each row and column; each entry must be a unit of $R^{+}$, and for $\left(k_{\alpha}\right) \in \oplus R,\left(k_{\alpha}\right) X^{t} \in K$ if and only if $\left(k_{\alpha}\right) \in K$. Then $G$ has the following action on $\oplus R / K$ defined by the map $a: G \times(\oplus R / K) \rightarrow \bigoplus R / K, a\left(X,\left(r_{\alpha}\right)+K\right)=\left(r_{\alpha}\right) X^{t}+K$. It is easy to show that for each $X$ in $G$, the map $a(X, \ldots)$ is an $R$-module automorphism of $M$ and that $a(X, \ldots) P \subseteq P$ so $a(X, \ldots)$ is order preserving. Clearly the inverse of $a(X, \ldots)$ is order preserving. If a: $G \rightarrow A$ by $\mathrm{a}(X)=a(X, \ldots)$ then $\mathrm{a}(X Y)=\mathrm{a}(X) \mathrm{a}(Y)$ and $\mathrm{a}$ is a homomorphism.

THEOREM 5. G/ker a is isomorphic to $A$ the group of order-preserving $R$-module automorphisms of $\left(M, M^{+}\right)$.

Proof. We need only show that a is onto $A$. If $g \in A$ then $g$ preserves $R^{+}$-bases. By Theorems 1 and 2 there are units $u_{\alpha}$ of $R^{+}$and a permutation 
$\sigma$ of $\kappa$ such that $g\left(i_{\alpha}(1)+K\right)=u_{\alpha} i_{\sigma(\alpha)}(1)+K$. Recall that $\left\{i_{\alpha}(1)+K \mid \alpha<\right.$ $\kappa\}$ is an $R^{+}$-basis for $P$. Since $g$ is an $R$-module homomorphism,

$$
g\left(\left(r_{\alpha}\right)+K\right)=\sum r_{\alpha} g\left(i_{\alpha}(1)\right)+K=\sum r_{\alpha} u_{\alpha} i_{\sigma(\alpha)}(1)+K .
$$

Define $X$ in $G$ by $X_{\beta \alpha}=0$ unless $\beta=\sigma(\alpha)$ in which case $X_{\sigma(\alpha) \alpha}=u_{\alpha}$. Then

$$
\left(r_{\alpha}\right) X^{t}+K=\sum r_{\alpha} u_{\alpha} i_{\sigma(\alpha)}(1)+K=g\left(\left(r_{\alpha}\right)+K\right) .
$$

Hence $a(X, \ldots)=g$ and $a$ is onto.

We remark that ker $\mathbf{a}=\left\{X \in G \mid i_{\alpha}\left(X_{\alpha \alpha}\right)+K=i_{\alpha}(1)+K\right.$ for all $\left.\alpha<\kappa\right\}$. For if $\mathrm{a}(X, \ldots)=1$ then in particular $\mathrm{a}\left(X, i_{\alpha}(1)+K\right)=i_{\alpha}(1)+K$. Suppose $\sigma$ is the permutation determining $X\left(X_{\beta \alpha}=0\right.$ unless $\beta=\sigma(\alpha)$ and $X_{\sigma(\alpha) \alpha}$ is a unit of $\left.R^{+}\right)$. Then $\mathrm{a}\left(X, i_{\alpha}(1)+K\right)=X_{\alpha \sigma(\alpha)} i_{\sigma(\alpha)}(1)+K=i_{\alpha}(1)+K$ and $R^{+}$-independence requires $\sigma(\alpha)=\alpha$. So $\sigma=1$ and $X_{\alpha \alpha} i_{\alpha}(1)+K=i_{\alpha}(1)+$ $K$. Conversely if $X$ is diagonal and $X_{\alpha \alpha} i_{\alpha}(1)+K=i_{\alpha}(1)+K$ it is clear that $X \in$ ker a.

There are several conditions which imply that ker $a=\{1\}$ in $G$. For example if $\left(R, R^{+}\right)$is totally ordered then ker $\mathrm{a}=\{1\}$. For $X_{\alpha \alpha} i_{\alpha}(1)+K=$ $i_{\alpha}(1)+K$ implies $\left(X_{\alpha \alpha}-1\right)\left(i_{\alpha}(1)+K\right)=0$ and as $X_{\alpha \alpha}-1$ or $1-X_{\alpha \alpha} \in$ $R^{+}$the strictness of the order gives $X_{\alpha \alpha}=1$.

4. Examples of nonexistence of $R$-bases. As expected not every $\left(R, R^{+}\right)$module $\left(M, M^{+}\right)$has an $R^{+}$-basis for its cone $M^{+}$.

Lemma 2. Let $\left(M, M^{+}\right)$be an $\left(R, R^{+}\right)$-module, $m_{1}, m \in M^{+}$and $r \in R^{+}$. If $m_{1}=r m$ and $r$ is not a unit of $R^{+}$then any set $B \subseteq M^{+}$containing $m_{1}$ is not an $R^{+}$-basis for a subcone of $M^{+}$which contains $m$.

Proof. Let $B \subseteq M^{+}$contain $m_{1}$ and suppose $R^{+} B$ contains $m$. Assuming $m_{1}=r m$ we show that $B$ is not $R^{+}$-independent. Let $m=\Sigma r_{i} b_{i}$ with $r_{i}$ in $R^{+}$ and $b_{i}$ in $B$. So $m_{1}=\sum r r_{i} b_{i}$ and as $m_{1} \in B$, if $B$ is $R^{+}$-independent, $m_{1}=b_{j}$ and $b_{j}$ appears on the right of this sum. If $<'$ is the order induced on $M$ by the cone $R^{+} B$ then $\sum_{i \neq j} r r_{i} b_{i}=\left(1-r r_{j}\right) b_{j}<^{\prime} b_{j}$. By hypothesis $1-r r_{j} \neq 0$ so $\left(1-r r_{j}\right) b_{j}$ is strictly positive in $R^{+} B$. Thus $B$ is not $R^{+}$-independent.

Corollary 1. Let $\left(G, G^{+}\right)$be a totally ordered divisible abelian group. Then $G^{+}$has no $Z^{+}$-basis.

Proor. Let $g \in G^{+}$, then $g / 2 \in G^{+}$. So $g$ is not in any $Z^{+}$-basis for $G^{+}$. So $G^{+}$has no $Z^{+}$-basis.

In particular $\mathbf{R}^{+}$and $Q^{+}$do not have $Z^{+}$-bases.

LEMMA 3. Let $\left(F, F^{+}\right)$be a totally ordered field which is also an $\left(R, R^{+}\right)$module. Let $R^{+} \subseteq F^{+}$and $\zeta^{\prime}$ be the order on $F$ induced by $F^{+}$. If for every nonzero $f$ in $F^{+}$there is an $r$ in $R^{+}$with $0<^{\prime} r<^{\prime} f$ then $F^{+}$is of degree 1 over $R^{+}$or $F^{+}$has no $R^{+}$-basis. 
Proof. For every $f$ in $F^{+} \backslash\{0\},\{f\}$ is an $R^{+}$.basis for the cone $R^{+} f \subseteq F^{+}$. Now suppose $F^{+}$has $R^{+}$-basis $B$ and let $b, c$ be distinct members of $B$. Since $1 \in F^{+}$and $F$ is totally ordered, if $c^{-1}<{ }^{\prime} 0$ then $1<{ }^{\prime} 0$, a contradiction of Lemma 1. So $c^{-1} \in F^{+}$. Choose $r \in R^{+}$such that $0<^{\prime} r \leqslant{ }^{\prime} b c^{-1}$ so $0<<^{\prime} b$ - $r c$ and for $b_{i}$ in $B$ and $r_{i}$ in $R^{+}, b=r c+\sum r_{i} b_{i}$. By $R^{+}$-independence of $B, b=b_{j}$ appears on the right in this sum. So

$$
0<<^{\prime}\left(1-r_{j}\right) b=r c+\sum_{i \neq j} r_{i} b_{i}<^{\prime} b .
$$

This contradicts $R^{+}$-independence.

Corollary 2. $\mathbf{R}^{+}$has no $Q^{+}$-basis.

\section{BIBLIOGRAPHY}

1. M. N. Bleicher and E. Marczewski, Remarks on dependence relations and closure operators, Collog. Math. 9 (1962), 209-212. MR 26 \#58.

2. M. N. Bleicher and H. Schneider, The decomposition of cones in modules over ordered rings, J. Algebra 1 (1964), 233-258. MR 29 \#5866.

3. P. M. Cohn, Universal algebra, Harper and Row, New York, 1965. MR 31 \#224; erratum 32, p. 1754.

4. L. Fuchs, Partially ordered algebraic systems, Addison-Wesley, Reading, Mass., 1963. MR 30 \#2090.

5. G. A. Heuer, Discrete ordered rings, Fund. Math. 85 (1974), no. 2 121-138. MR 49 \#8915.

6. N. Jacobson, Lectures in abstract algebra. II, Van Nostrand, Princeton, N.J., 1953. MR 14, 837.

7. P. Ribenboim, On ordered modules, J. Reine Angew. Math. 225 (1967), 120-146. MR 34 \#5877. 21402

Department of Mathematics, United States Naval Academy, Annapolis, Maryland 